\title{
Prognostic impact of a tumor-infiltrating lymphocyte subtype in triple negative cancer of the breast
}

\author{
Tsengelmaa Jamiyan ${ }^{1,5} \cdot$ Hajime Kuroda $^{1} \cdot$ Rin Yamaguchi $^{2} \cdot$ Yoshimasa Nakazato $^{1} \cdot$ Shuhei Noda ${ }^{1}$.

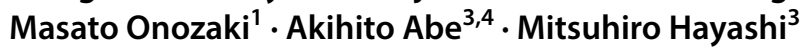

Received: 6 January 2020 / Accepted: 20 March 2020 / Published online: 28 March 2020

(c) The Author(s) 2020

\begin{abstract}
Background Tumor-infiltrating lymphocytes (TILs) have recently been reported as an important factor in the tumor microenvironment and influence the growth and progression of cancer. However, the relationship between immune cell subpopulations, such as CD4+, CD8+, and FOXP3+, in breast cancer, especially in triple negative carcinoma (TNC), remains unclear. Methods The subjects were 107 patients with TNC that were surgically resected at Dokkyo Medical University Hospital between 2006 and 2018. The expression of CD4+, CD8+, and FOXP3+ was evaluated in TILs and expressed as the numbers of positive cells.

Results Univariate analysis revealed that the TILs were not prognostically significant. In multivariate analyses, increased infiltration of intratumoral (i) CD4+ TILs was found to have a good prognosis in relapse-free survival (RFS). In contrast, a high stromal CD8+ TILs level was found to be a favorable prognostic factor in RFS $(p=0.038)$ and overall survival (OS) $(p=0.046)$. A low sFOXP3 + TILs level was significantly associated with favorable RFS $(p<0.001)$ and OS $(p=0.029)$.

Conclusions The present study demonstrated no difference in TILs and survival in TNC. However, there was a significant correlation in prognosis with levels of iCD4+, sCD8+, and sFOXP3 + TILs in TNC. The difference in TNC clinical outcome may be due to the subtype of the infiltrating TILs.
\end{abstract}

Keywords Breast $\cdot$ Triple negative cancer $\cdot$ CD $4 \cdot$ CD $8 \cdot$ FOXP3

\section{Introduction}

Gene expression profiling studies have divided invasive breast cancer into several major subtypes [1]. The so-called 'triple negative carcinoma' (TNC) is characterized by a lack of expression of the estrogen receptor (ER) and progesterone

Hajime Kuroda

hajimek@dokkyomed.ac.jp

1 Department of Diagnostic Pathology, Dokkyo Medical University, 880 Kitakobayashi, Mibu, Tochigi 321-0293, Japan

2 Department of Pathology \& Laboratory Medicine, Kurume University Medical Center, Kurume, Japan

3 Breast Center, Dokkyo Medical University, Dokkyo, Japan

4 Department of Surgery II, Dokkyo Medical University, Dokkyo, Japan

5 Department of Pathology and Forensic Medicine, Mongolian National University of Medical Sciences, Ulan Bator, Mongolia receptor (PgR), and absence of human epidermal growth factor receptor 2 (HER2) protein overexpression; this type is known to have a poor prognosis [2,3]. Tumor-infiltrating lymphocytes (TILs) have recently been reported as an important factor in the tumor microenvironment and influence the growth and progression of cancer.

The majority of TILs in cancer are of the T-cell phenotype, which includes CD4+ (helper cells) and CD8+ (cytotoxic cells) lymphocytes. CD4 $+\mathrm{T}$ lymphocytes are important for priming tumor-specific CD8 + TILs as well as for the secondary expansion and memory of CD8 + TILs [4]. Furthermore, many immunohistochemical studies have concluded that CD8 + TILs have antitumor activity as evaluated by the favorable prognosis in colorectal [5], ovarian [6], esophageal [7], renal [8], lung [9], and pancreatic [10] tumors. However, the impact of CD8 + TILs in breast cancer is controversial. Previous breast cancer studies have reported that marked infiltration of CD8 + TILs is associated with good prognosis, while several studies have found a negative correlation or no correlation with prognosis [11-14]. 
Therefore, the assumption that lymphocyte infiltration promotes or prevents cancer cannot be confirmed without clarifying which immune cell phenotype is involved.

The role of CD4 + TILs in immune activity has been reported in many cancer patients. However, the discovery of regulatory T cells (Treg) has markedly changed conventional speculation regarding the role of $\mathrm{CD} 4+\mathrm{T}$ lymphocytes in anti-tumor immunity.

Forkhead box protein 3 (FOXP3) plays a critical role in the generation of immune-suppressive CD4 + Tregs, and this leads to immune tolerance of CD8+ killer cells [15]. Excess FOXP3 expression leads to Treg proliferation and severe immunodeficiency, whereas lack of FOXP3 results in immune system activation and aggressive lymphoproliferation. Furthermore, FOXP3 is involved in immune escape mechanisms and both poor survival and improved survival in breast cancer have been reported [15-17]. There have been several reports on TILs in breast cancer [18, 19]. However, the relationship between immune cells subpopulations, such as $\mathrm{CD} 4+, \mathrm{CD} 8+$ and $\mathrm{FOXP} 3+$, in breast cancer, especially in TNC, remains unclear. We herein discuss the clinicopathological features and possible roles of immune cells in TNC.

\section{Materials and methods}

\section{Patients}

The subjects were 107 patients with TNC, which was surgically resected at Dokkyo Medical University Hospital between 2006 and 2018. Patients' clinical information was retrieved from institutional medical records. Clinical outcome was also documented. For each case, all available hematoxylin and eosin-stained whole-tissue sections were reviewed to confirm the diagnosis of mammary disease with no knowledge of prior histological results or clinical outcomes. The present study was approved by the Ethics Committees of Dokkyo Medical University (Tochigi, Japan; registration number 28009).

\section{Immunohistochemistry (IHC)}

Surgical sections were immunostained for ER (clone SP1, Novocastra (Leica), prediluted, nuclear), PgR (clone 1E2, Novocastra (Leica), prediluted, nuclear), HER2 (clone 4B5, Roche (VENTANA), prediluted, membranous), CD4 (CD4, clone 1F6, Novocastra (Leica), 1:40), CD8 (CD8, clone 4B11, Novocastra (Leica), prediluted) and FOXP3 (FOXP3, clone 236A/E7, abcam, 1:50). Counterstaining was performed with hematoxylin. ER and PgR status were considered positive if any positive cells were detected within the tumor. HER2 status was assessed according to the guidelines defined by the American Society of Clinical Oncology/College of American Pathologists [20]. We estimated the TILs on hematoxylin and eosin (H\&E) stained sections according to the criteria proposed by the International ImmunoOncology Biomarkers Working Group [21]. Lymphocytes in contact with or within the tumor epithelium were defined as intratumoral (i), whereas lymphocytes in the interstitial space or in the stromal areas were defined as stromal (s). TILs were defined as all mononuclear cells, including lymphocytes, within the stromal area, and excluded necrosis, crush artifacts, regressive hyalinization, as well as granulocytes and other polymorphonuclear leukocytes (Fig. 1). TILs levels were categorized as high $(\geq 30 \%)$ and low $(<30 \%)$ adopting previously validated cut-offs [22]. The expressions of CD4, CD8, and FOXP3 were evaluated in TILs and expressed as the numbers of positive cells counted in each case at $\times 400$ magnification ( $\times 40$ objective) (Fig. 1). For statistical analyses, the number of positive cells was divided into lower and higher groups based on cut-off points according to the median. As a result, the cut-off for iCD4+ was 3, sCD4+ was 54, iCD8+ was 7 , sCD8+ was 43 , iFOXP3+ was 3 , and sFOXP3+ was 32 . All sections were evaluated by two pathologists (TJ and HK) who had no previous knowledge of the patients' clinical information, and the results were averaged.

\section{Statistical analysis}

The associations between CD4+, CD8+, and FOXP3 + TILs and clinicopathological variables were examined by $x^{2}$-test. Relapse-free survival (RFS) was defined as the time from surgery to recurrence, including metastatic disease. Overall survival (OS) was determined from the date of surgery to the date of death by cancer or to the date of the last follow-up. For significance testing in Kaplan-Meier survival analysis, we used the log-rank test. The hazard ratios (HRs) and 95\% confident intervals (CIs) were calculated by Cox proportional hazard models. Multivariate Cox regression analysis including all potential variables that were significantly associated with survival in each univariate analysis was performed. All statistical tests were considered significant at the $p<0.05$ level. Statistical analysis was performed using IBM SPSS Statistics 25 (IBM, Armonk, NY, United States).

\section{Results}

Clinicopathological findings and expression of immune markers $(\mathrm{CD} 4+, \mathrm{CD} 8+, \mathrm{FOXP} 3+)$ are summarized in Tables 1 and 2, respectively. The patient ages ranged from 28 to 89 years, with a mean of 58.9 years. Tumor size ranged from 0.3 to $10.0 \mathrm{~cm} ; 64.5 \%$ were $\leq 2.0 \mathrm{~cm}$ and $35.5 \%$ were $>2.0 \mathrm{~cm}$ in diameter. They presented with histological 
Fig. 1 Triple-negative carcinoma of the breast. a Representative H\&E staining images of iTILs and sTILs. b-d Representative images of immunohistochemical staining of low and high CD4+, CD8+ and FOXP3 + TILs infiltration densities in intratumoral and stromal areas. $\mathbf{b}$ CD4 + TILs, c CD8 + TILs, and $\mathbf{d}$ FOXP3 + TILs. Original magnification: $\times 400$

A

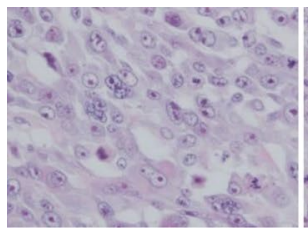

Low iTILs

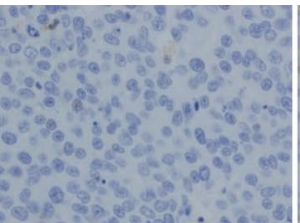

Low iCD4

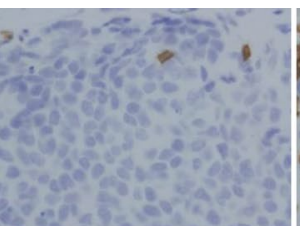

Low iCD8

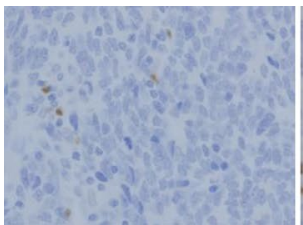

Low iFOXP3

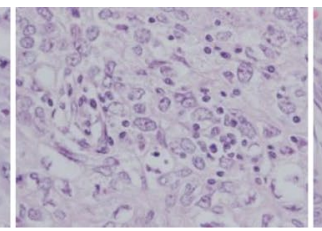

High iTILs

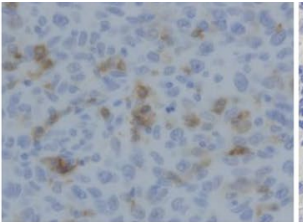

High iCD4

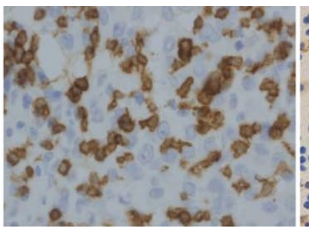

High iCD8

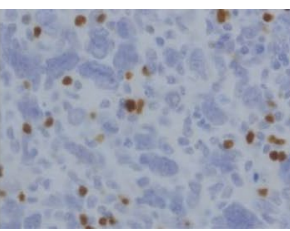

High iFOXP3

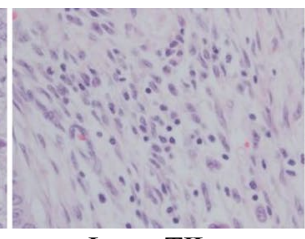

Low sTILs

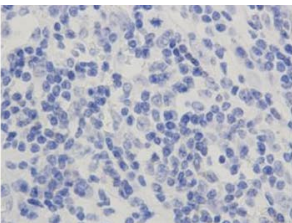

Low sCD4

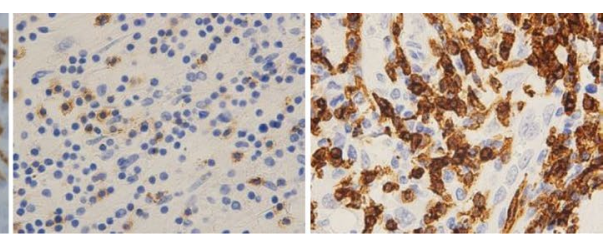

Low sCD8

High sCD8

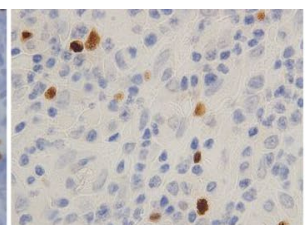

Low sFOXP3

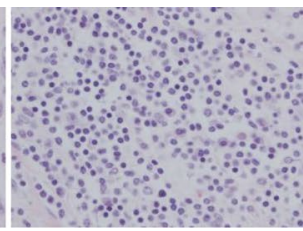

High sTILs

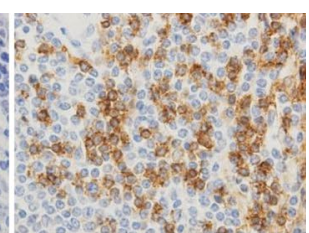

High sCD4 high grade (76/107) and lymph node metastasis (30/107). Recurrence occurred in 19 (19.2\%) of 99 patients, and cancer-associated death occurred in $12(12.1 \%)$ of 99 patients. Median follow-up for the assessment of RFS was 42.13 (0-120) months and that for overall survival was 43.86 (0-120) months. The high expression of iCD4 + TILs was associated with larger tumor size $(p=0.034)$ and higher histological grade $(p=0.006)$. Lymph node status $(p=0.036$, $p=0.05)$ and expression of CD8 + TILs in both $i$ and $s$ areas had a significant correlation in the $x^{2}$-test.

\section{Univariate and multivariate Cox regression analysis correlation with CD4, CD8, and FOXP3 levels with RFS and OS}

Univariate and multivariate Cox regression analysis of RFS and OS were performed using clinicopathological findings and expression of TILs (Tables 2, 3). Univariate analysis revealed that the conventional clinicohistological tumor parameters, including age, tumor size, histological grade, lymph node status, and TILs were not prognostically significant. Furthermore, the iCD4 + TILs demonstrated a significant association with RFS ( $p=0.044)$, but no significant difference in terms of OS ( $p=0.074)$. Both in the RFS and OS, sCD4 + TILs patients showed no significance in univariate analysis $(p=0.261 ; p=0.254)$. However, the expressions of sCD8 + and sFOXP3 + TILs were associated with RFS and $\mathrm{OS}(p=0.020 ; p=0.032)$. The $\mathrm{iCD} 4+, \mathrm{sCD} 8+$ and sFOXP3 + TILs found to have significant prognostic value in univariate analysis were selected for Cox proportional hazard analyses and the significance of their prognostic association was confirmed by multivariate assessment. In multivariate analyses, patients with high expression of iCD4 + TILs had a significantly longer RFS (HR 0.172, 95\% CI 0.037-0.792, $p=0.024)$. Increased infiltration of SCD8 + TILs was found to be a favorable prognostic factor in RFS (HR 0.225, 95\% CI $0.061-0.836, p=0.026)$ and OS (HR $0.263,95 \% \mathrm{CI}$ $0.071-0.975, p=0.046)$. In contrast, a low sFOXP3 + TILs level was found to be significantly associated with favorable RFS (HR 7.426, 95\% CI 1.596-34.552, $p<0.011$ ) and OS (HR 5.467, 95\% CI 1.192-25.07, $p=0.029$ ) (Table 4).

Moreover, the sCD4/CD8, sCD8/FOXP3, and sFOXP3/ CD4 ratios were significantly associated with both RFS and OS in univariate analysis. We investigated these variables for their independent association with RFS and OS using a multivariate Cox regression model. The results revealed that sCD8/FOXP3 had prognostic significance for RFS (HR 0.130, 95\% CI 0.025-0.669, $p=0.015$ ) and OS (HR $0.157,95 \%$ CI $0.031-0.797, p=0.026)$. The sFOXP3/CD4 ratio was also significantly associated with RFS (HR 2.766, 


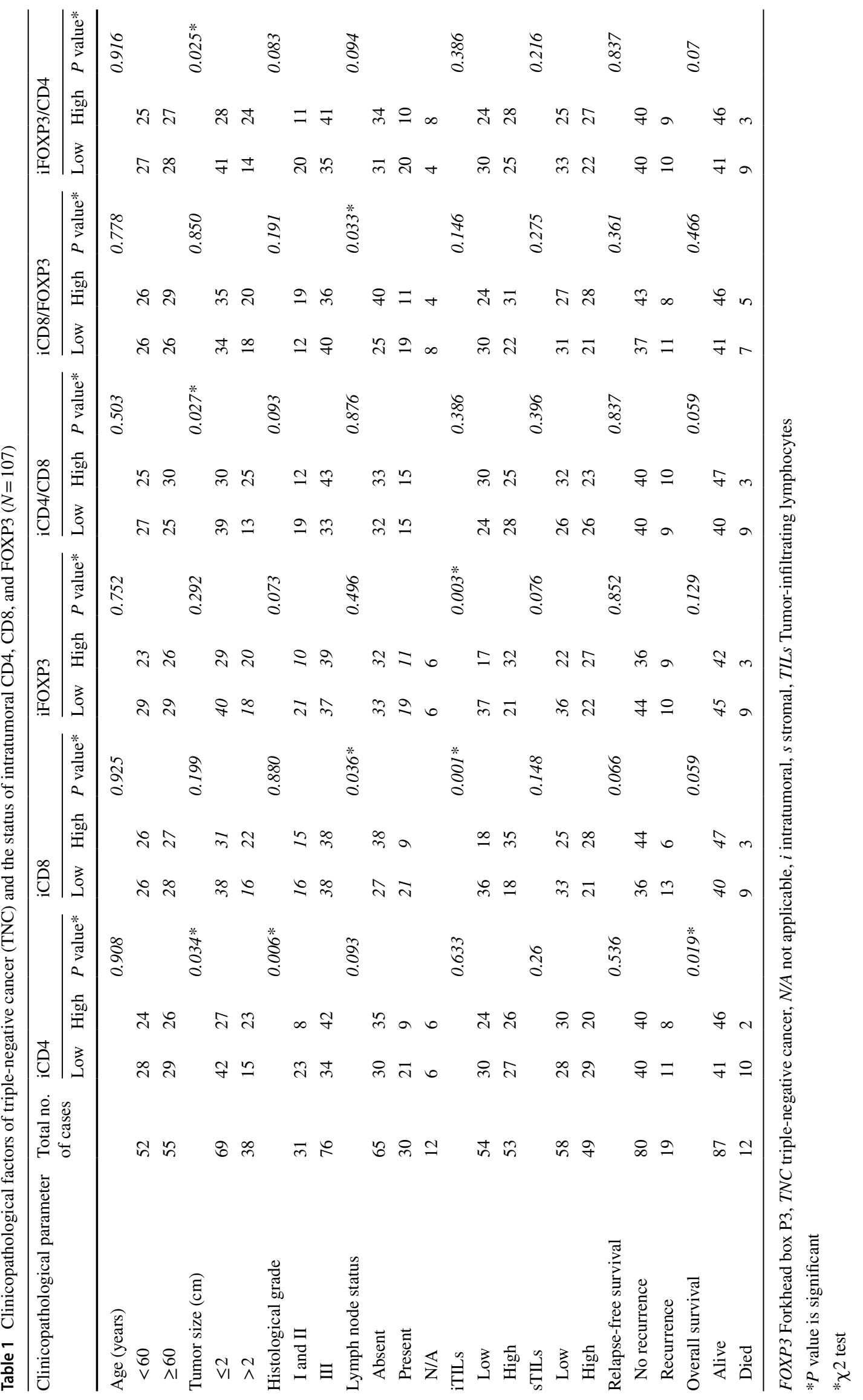




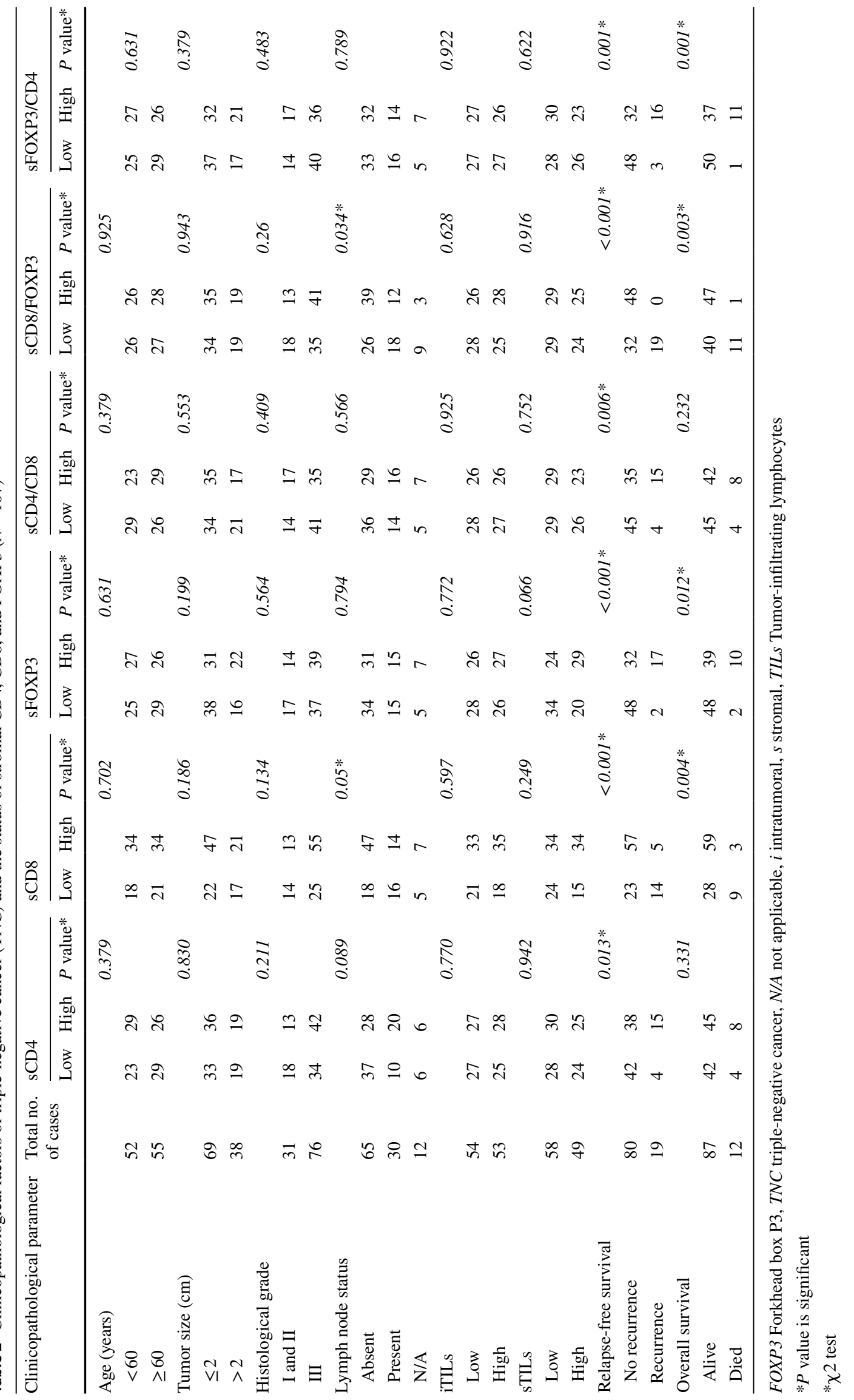


Table 3 Hazards for triple-negative cancer (TNC) relapse-free survival (RFS) in the entire cohort with univariate and multivariate analyses

\begin{tabular}{|c|c|c|c|c|c|c|}
\hline \multirow[t]{2}{*}{ Clinicopathological feature } & \multicolumn{3}{|c|}{ Univariate analysis } & \multicolumn{3}{|c|}{ Multivariate analysis } \\
\hline & HR & $95.0 \% \mathrm{CI}$ & $P$ value* & HR & $95.0 \% \mathrm{CI}$ & $P$ value* \\
\hline Age $(<60$ vs. $\geq 60)$ & 0.323 & $0.087-1.193$ & 0.090 & & & \\
\hline Tumor size $(2 \mathrm{~cm}$ vs. $>2 \mathrm{~cm})$ & 2.527 & $0.807-7.915$ & 0.111 & & & \\
\hline Histological grade (I, II vs. III) & 2.149 & $0.520-8.871$ & 0.290 & & & \\
\hline Lymph node status (absent vs. present) & 1.678 & $0.815-3.455$ & 0.160 & & & \\
\hline iTILs (high vs. low) & 0.584 & $0.185-1.846$ & 0.360 & & & \\
\hline sTILs (high vs. low) & 0.499 & $0.150-1.659$ & 0.257 & & & \\
\hline iCD4 (low vs. high) & 0.210 & $0.046-0.959$ & $0.044 *$ & 0.172 & $0.037-0.792$ & $0.024 *$ \\
\hline sCD4 (low vs. high) & 1.992 & $0.599-6.626$ & 0.261 & & & \\
\hline iCD8 (low vs. high) & 0.303 & $0.0082-1.119$ & 0.073 & & & \\
\hline sCD8 (low vs. high) & 0.213 & $0.058-0.785$ & $0.020^{*}$ & 0.225 & $0.061-0.836$ & $0.026^{*}$ \\
\hline iFOXP3 (low vs. high) & 0.333 & $0.090-1.230$ & 0.099 & & & \\
\hline sFOXP3 (low vs. high) & 5.804 & $1.265-26.62$ & $0.024 *$ & 7.426 & $1.596-34.552$ & $0.011^{*}$ \\
\hline \multicolumn{7}{|l|}{ Ratio of immune cells } \\
\hline iCD4/CD8 & 0.369 & $0.100-1.365$ & 0.135 & & & \\
\hline sCD4/CD8 & 1.362 & $1.049-1.769$ & $0.021 *$ & 1.003 & $0.650-1.549$ & 0.988 \\
\hline iCD8/FOXP3 & 0.626 & $0.199-1.973$ & 0.424 & & & \\
\hline sCD8/FOXP3 & 0.200 & $0.065-0.616$ & $0.005 *$ & 0.130 & $0.025-0.669$ & $0.015^{*}$ \\
\hline iFOXP3/CD4 & 0.356 & $0.096-1.314$ & 0.121 & & & \\
\hline sFOXP3/CD4 & 1.858 & $1.160-2.977$ & $0.010^{*}$ & 2.766 & $1.443-5.302$ & $0.002 *$ \\
\hline
\end{tabular}

Multivariate cox regression analyses were performed for all potential variables that were significantly associated with survival in univariate analysis $R F S$ recurrence-free survival. TNC triple-negative cancer, $H R$ hazard ratio, $C I$ confidence interval, i intratumoral, s stromal, TILs tumorinfiltrating

lymphocytes, FOXP3 Forkhead box P3

${ }^{*} P$ value is significant

95\% CI $1.443-5.302, p=0.002)$ and OS (HR 3.386, 95\% CI $1.684-6.807, p=0.001)$.

We investigated survival with regard to the different expressions of CD4 + TILs, CD8 + TILs FOXP3 + TILs status using the Kaplan-Meier method and log-rank test. Patients with high expression of iCD4 + TILs had significantly longer RFS $(p=0.026)$ and OS $(p=0.038)$ than those with low expressions of iCD4 + TILs (Fig. 2a). The expressions of iCD8 + TILs, and iFOXP3 + TILs, were not related to either RFS $(p=0.057, p=0.082)$ or OS $(p=0.058$, $p=0.060$, respectively; Fig. 2a). sCD4 + TILs were not significantly correlated with OS $(p=0.244)$ or RFS $(p=0.253)$ in patients with TNC (Fig. 2b). In contrast, a high number of sCD8+ and low number of sFOXP3 + TILs were significantly correlated with favorable RFS $(p=0.010$; $p=0.010)$ and $\mathrm{OS}(p=0.019 ; p=0.009$, respectively; Fig. 2b). Kaplan-Meier analysis revealed survival differences based on the ratio between $i$ and $s$ infiltration of immune cells (CD4/CD8, CD8/FOXP3, FOXP3/CD4). We observed no significant difference in the ratios of $i$ immune cells (CD4/CD8, CD8/FOXP3, FOXP3/CD4) between RFS $(p=0.118, p=0.418, p=0.104)$ and OS $(p=0.171$, $p=0.408, p=0.102$ ) (Fig. 2c). With regard to the ratio of immune cells, no significant association was seen between the $\mathrm{sCD} 4 / \mathrm{CD} 8$ ratio and RFS or OS ( $p=0.327 ; p=0.423)$. Patients with greater changes in the sCD8/FOXP3 ratio had significantly better RFS and OS compared with those with smaller changes $(p=0.006 ; p=0.011)$ (Fig. $2 \mathrm{~d})$. Furthermore, we found that patients with a high sFOXP3/CD4 ratio had a significantly poorer RFS and OS $(p=0.002 ; p=0.002)$ (Fig. 2d).

\section{Discussion}

The characteristic features of TNC are large anaplastic cells and poor prognosis. Invasive carcinoma including medullary features with massive TILs has a better prognosis than the typical types of invasive mammary carcinomas [3, 23]. Furthermore, a recent report suggested the prognostic importance of TILs in high-grade breast cancers. Kurozumi et al. recently investigated the relationship between TILs and prognosis in 294 cases and reported that high stromal TILs expression was a good prognostic marker in ER-negative cancers [24]. Ibrahim et al. also demonstrated that TILs were significantly correlated with a favorable breast cancer 
Table 4 Hazards for triple-negative cancer (TNC) overall survival (OS) in the entire cohort with univariate and multivariate analyses

\begin{tabular}{|c|c|c|c|c|c|c|}
\hline Clinicopathological feature & HR & $\begin{array}{l}\text { Univariate analysis } \\
95.0 \% \mathrm{CI}\end{array}$ & $P$ value* & HR & $\begin{array}{l}\text { Multivariate analysis } \\
95.0 \% \text { CI }\end{array}$ & $P$ value* \\
\hline Age (<60 vs. $\geq 60)$ & 0.316 & 0.08501 .169 & 0.084 & & & \\
\hline Tumor size $(2 \mathrm{~cm}$ vs. $>2 \mathrm{~cm})$ & 3.012 & $0.962-9.431$ & 0.058 & & & \\
\hline Histological grade (I, II vs. III) & 2.161 & $0.472-9.886$ & 0.321 & & & \\
\hline Lymph node status (absent vs. present) & 1.820 & $0.868-1.169$ & 0.113 & & & \\
\hline TILs (high vs. low) & 0.476 & $0.143-1.585$ & 0.226 & & & \\
\hline iCD4 (low vs. high) & 0.231 & $0.051-1.055$ & 0.059 & & & \\
\hline sCD4 (low vs. high) & 2.014 & $0.604-6.712$ & 0.254 & & & \\
\hline iCD8 (low vs. high) & 0.304 & $0.082-1.124$ & 0.074 & & & \\
\hline sCD8 (low vs. high) & 0.239 & $0.065-0.885$ & $0.032 *$ & 0.263 & $0.071-0.975$ & $0.046^{*}$ \\
\hline iFOXP3 (low vs. high) & 0.305 & $0.082-1.133$ & 0.076 & & & \\
\hline sFOXP3 (low vs. high) & 5.944 & $1.298-27.22$ & $0.022 *$ & 5.467 & $1.192-25.07$ & $0.029 *$ \\
\hline \multicolumn{7}{|l|}{ Ratio of immune cells } \\
\hline iCD4/CD8 & 0.413 & $0.111-1.530$ & 0.186 & & & \\
\hline sCD4/CD8 & 1.368 & $1.042-1.797$ & $0.024 *$ & 1.059 & $0.673-1.665$ & 0.804 \\
\hline iCD8/FOXP3 & 0.619 & $0.196-1.953$ & 0.414 & & & \\
\hline sCD8/FOXP3 & 0.215 & $0.070-0.661$ & $0.007 *$ & 0.157 & $0.031-0.797$ & $0.026^{*}$ \\
\hline iFOXP3/CD4 & 0.354 & $0.096-1.307$ & 0.119 & & & \\
\hline sFOXP3/CD4 & 2.224 & $1.347-3.669$ & $0.002 *$ & 3.386 & $1.684-6.807$ & $0.001 *$ \\
\hline
\end{tabular}

Multivariate cox regression analyses were performed for all potential variables that were significantly associated with survival in univariate analysis $O S$ overall survival. TNC triple-negative cancer, $H R$ hazard ratio, $C I$ confidence interval, i intratumoral, s stromal, TILs tumor-infiltrating lymphocytes, FOXP3 Forkhead box P3

*The $P$ value is significant

outcome in ER-negative tumors using meta-analysis including data on 2,987 patients [25]. However, we could not find significant differences in either iTILs or sTILs regarding the prognosis in TNC. Therefore, it is difficult to reach a conclusion regarding the prognosis of breast cancer based only on the TILs in TNC. Our results suggest that this prognosis in TNC is due, at least in part, to the presence of immune cell types that are closely associated with the tumor.

We found that TNC patients with a good prognosis had a predominance of sCD8 + TILs in both RFS and OS. Lymphocytes infiltrating a tumor indicate a local immune response and they play an important role in tumor progression $[11,12,26]$. The majority of infiltrating lymphocytes in tumors are CD8 + TILs and these have a cytotoxic effect [12, 27]. In several organs, high levels of CD 8 + TILs infiltration were associated with better prognosis [5-10]. In breast cancer, Ali et al. reported that $\mathrm{iCD} 8+$ and SCD8 + T cell infiltration was also associated with a significant reduction in the relative risk of death [11]. Furthermore, Liu et al. reported that iCD8 + and sCD8 + tumor-infiltrating lymphocytes are an independent prognostic factor associated with better survival in TNC [28]. Therefore, the greater predominance of CD8 + lymphocyte infiltration in TNC suggests that a strong immune response is occurring. However, we found increased infiltration of iCD4 + TILs was significantly associated with good prognosis only in RFS by multivariate analysis. In contrast, there have been a few reports examining the role of CD4 + TILs in breast cancers; they were associated with more aggressive behavior. Huang et al. reported that iCD4+ TILs negatively correlated with RFS in breast cancer [26]. Rubbert et al. reported a predominance of sCD4 + TILs among tumor-infiltrating lymphocytes in patients with larger tumors [29]. Furthermore, Macchetti et al. observed that in patients with lymph node metastasis, there was increased infiltration of sCD4 + TILs with a corresponding reduction in CD8 + cells [30]. Since CD4 + TILs are expressed in many $\mathrm{T}$ cell subsets including $\mathrm{T}$ helper 1 (Th1) cells, T helper 2 (Th2) cells and Tregs, each of these may have a different impact on prognosis. Th1 cells secrete several cytokines such as interferon gamma (IFNg), transforming growth factor beta (TGF $\beta)$, tumor necrosis factor alpha (TNF), and interleukin 2 (IL-2) [31]. These cytokines are involved in the function of CD8 + TILs and protect against tumor development and progression. In contrast, Th2 cells express several types of interleukin and induce loss of cytotoxicity [32]. Thus, CD4 + TILs that include many $\mathrm{T}$ cell subsets may explain why iCD4 + TILs was different in TNC.

In the present study, TNC patients with a good prognosis showed significantly lower expression of sFOXP3 + Tregs. Tregs are important mediators of immune tolerance that 

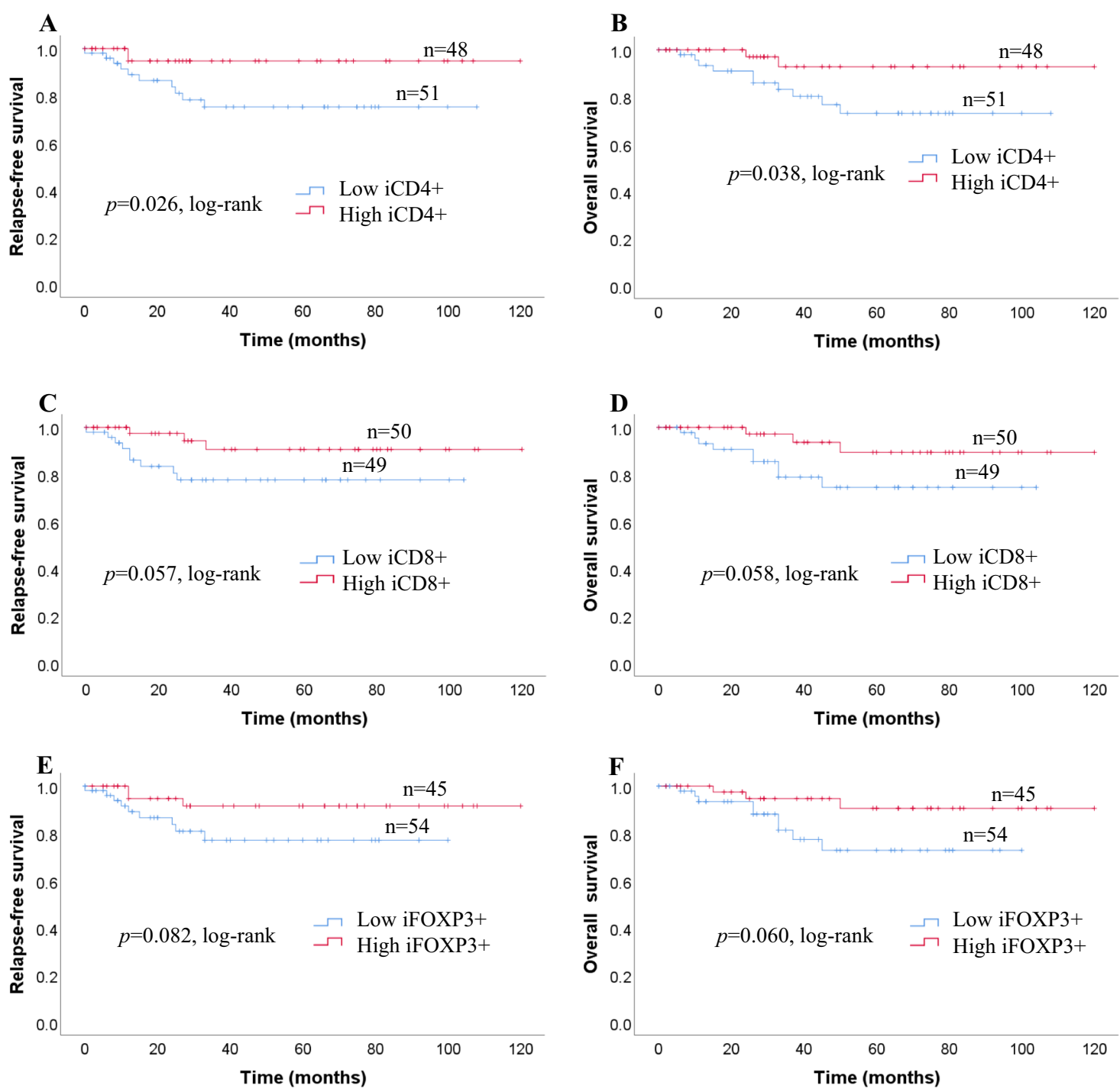

Fig. 2 a Recurrence-free survival (RFS) and overall survival (OS) in patients with iCD4+TILs, iCD8 + TILs, and iFOXP3+TILs. Estimated Kaplan-Meier curves of RFS (a) and OS (b) in patients with high or low iCD4+TILs, those of RFS (c) and OS (d) in patients with high or low iCD8 + TILs, and those of RFS (e) and OS (f) in patients with high or low iFOXP3 + TILs. b Prognostic significance of lymphocytic variables in breast cancer. Kaplan-Meier curves for overall survival (OS) and relapse-free survival (RFS) were stratified by the median values as the cut-off for prognostic evaluation and divided into low or high lymphocytic variable subsets. The blue solid line indicates patients with low values and the red solid line high values. sCD4+TILs did not demonstrate prognostic significance for

suppress $\mathrm{T}$ cell effects and inhibit immune-mediated tissue damage. FOXP3 is a member of the forkhead/wingedhelix family of transcription factors related to the regulation of the development and function of the immune system. Excess FOXP3 expression leads to Treg proliferation and severe immunodeficiency, whereas lack of FOXP3 results in immune system activation and aggressive
RFS (a) and OS (b), but high sCD8 + TILs was associated with both prolonged RFS (c) and OS (d). In contrast, high sFOXP3 + TILs was associated with both reduced RFS (e) and OS (f). c Kaplan-Meier survival curves illustrating the relapse-free survival (RFS) and overall survival (OS) according to the ratio of iCD4/CD8 (a, b), iCD8/ FOXP3 (c, d) and iFOXP3/CD4 (e, f). d Recurrence-free survival (RFS) and overall survival (OS) in patients with different sCD4/CD8, sCD8/FOXP3, and sFOXP3/CD4 ratios. Estimated Kaplan-Meier curves of RFS (a) and OS (b) in patients with high or low sCD4/ CD8 ratios, those of RFS (c) and OS (d) in patients with high or low sCD8/FOXP3 ratios, and those of RFS (e) and OS (f) in patients with high or low sFOXP3/CD4 ratios

lymphoproliferation [15, 16, 33]. FOXP3-expressing Tregs are reported to be abundant in tumor infiltrates and are involved in the immune escape mechanisms promoted by cancer. In several types of cancer, high levels of Tregs infiltration around the tumor were found to be correlated with poor prognosis [27, 34]. However, opinions vary among researchers regarding the role of FOXP3 + in breast cancer. 

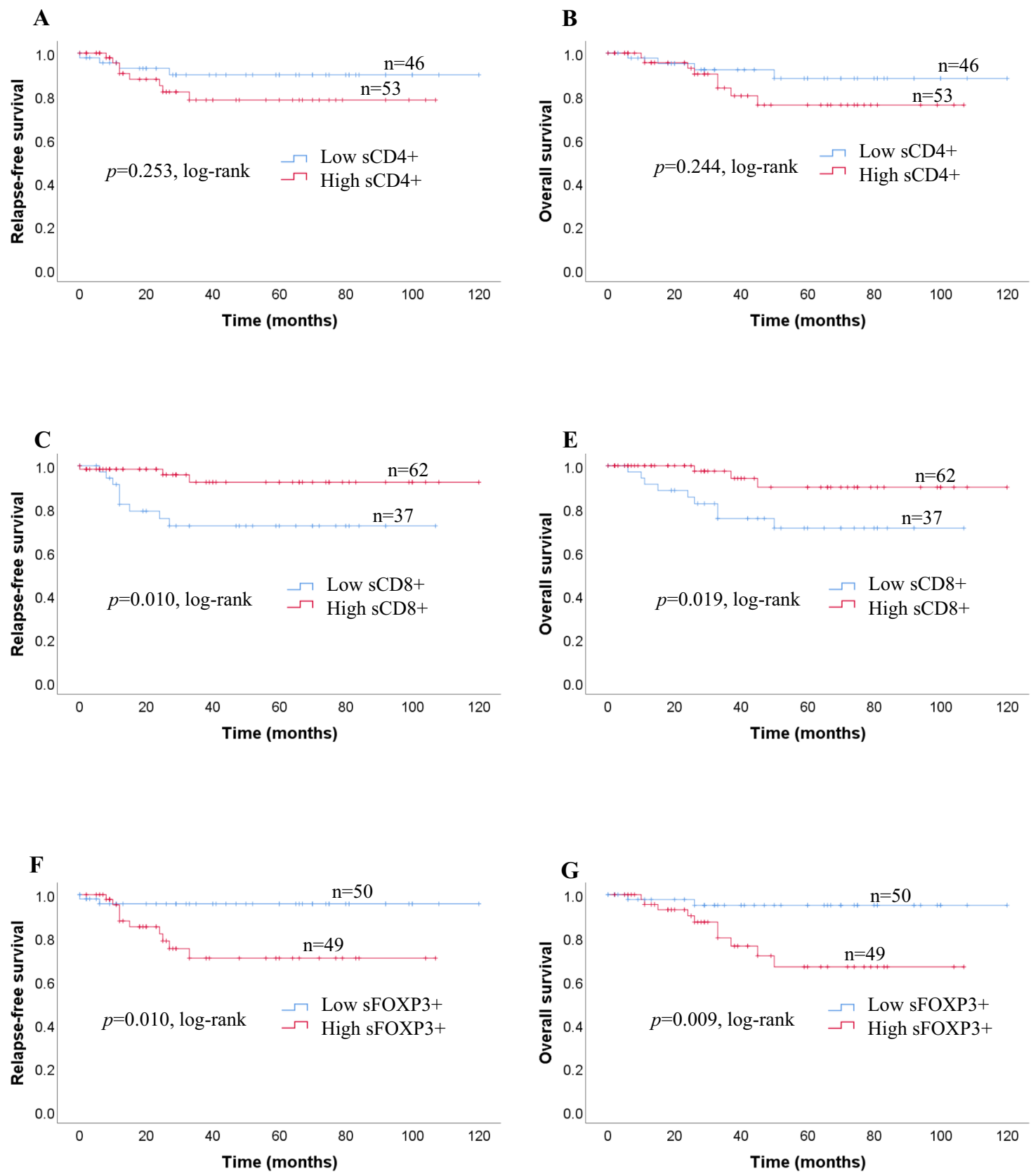

Fig. 2 (continued)

Castaneda et al. evaluated 98 TNC patients and higher expression of sFOXP3 + Tregs in TILs showed longer disease-free survival [35]. However, one limitation of this study is that they did not perform univariate and multivariate Cox regression analysis. In contrast, Kim et al. reported that higher numbers of FOXP3-expressing Tregs were associated with shorter RFS in breast cancers [36]. Furthermore, Peng et al. reported that high grade infiltrating ductal carcinoma with good prognosis showed significantly lower expression of FOXP3 [37]. In addition, a decreased ratio of CD8 + TILs to FOXP3 + Tregs infiltrating and surrounding tumors correlated with poor prognosis in breast cancer [38]. Thus our results are consistent with these findings that TNC patients with a good prognosis have lower expression of sFOXP3 + Tregs.

We also investigated the CD4/CD8, CD8/FOXP3, and FOXP3/CD4 ratio because there have been several studies that reported the CD8/FOXP3 ratio in breast cancer. Liu et al. reported an increased ratio of CD8/FOXP3 in the peritumoral area of non-luminal carcinoma and indicated 

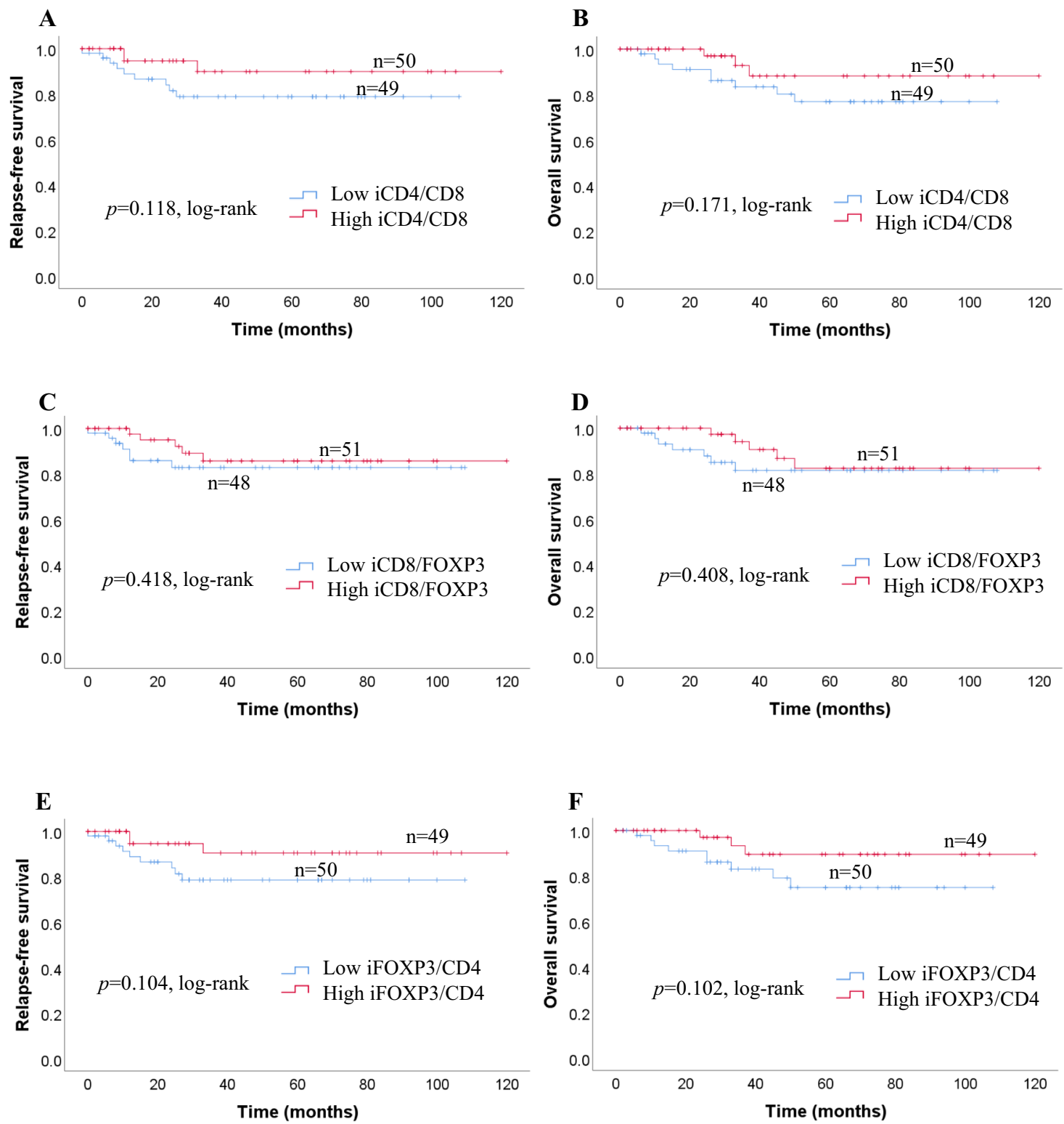

Fig. 2 (continued)

good survival of breast cancer [27]. A recent study by Miyashita et al. demonstrated that a high sCD8/FOXP3 ratio was associated with improved prognosis in TNC [39]. Furthermore, our study confirmed that not only sCD8/FOXP3, but also the sFOXP3/CD4 ratio, were significantly associated with both RFS and OS. It seemed that both FOXP3 and CD4 were associated with tumor progression, but FOXP3 was a stronger indicator. These results suggest that activation of cytotoxic TILs and Tregs may affect the clinical outcome.

\section{Conclusion}

The present study demonstrated no difference in either iTILs or sTILs and survival in TNC. However, we found higher numbers of iCD4 + TILs were significantly associated with good prognosis in RFS. Further, decreased sFOXP3 + TILs infiltrate and higher numbers of sCD8 + TILs in TNC were associated with a significantly good prognosis in both RFS and OS. Therefore, 
$\mathbf{A}$

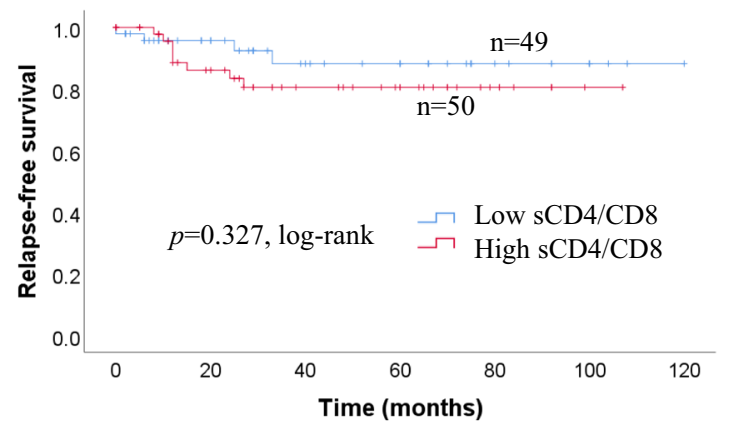

C

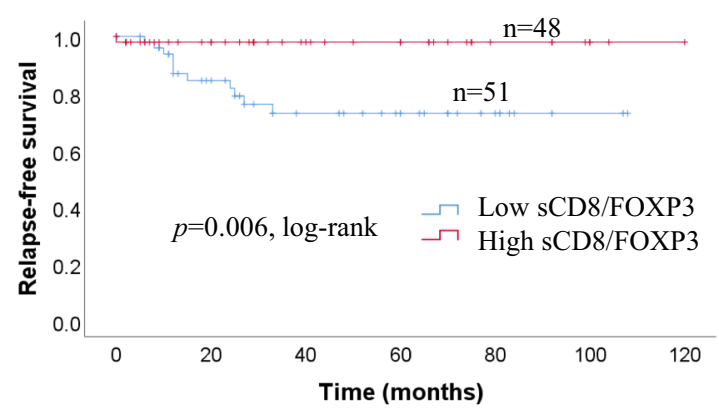

$\mathbf{F}$

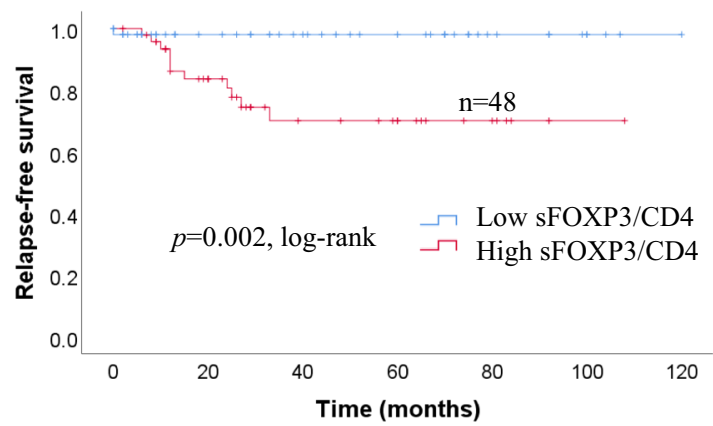

Fig. 2 (continued)

we should not simply focus on the TILs level in TNC. It is possible that a local immune response leading to killer cell expression occurs in some cases and suppression by regulating Tregs occurs in other cases. The difference in clinical outcome of TNC may be due to the subtype of the infiltrating TILs.

Acknowledgements The authors thank Dr Oi Harada, Chiaki Matsuyama and Ayako Shimizu for their advice and technical assistance with IHC staining.

\section{B}

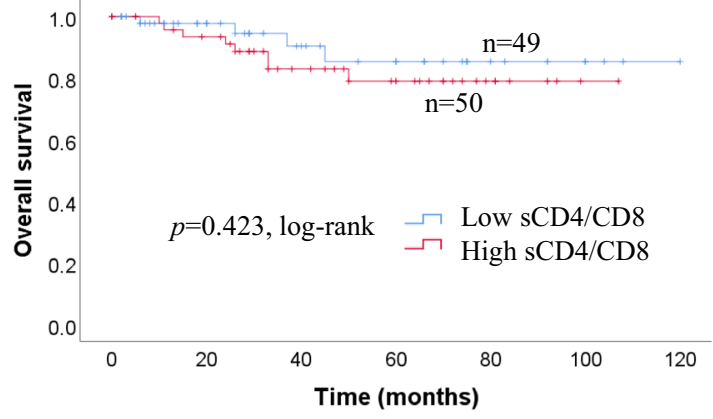

$\mathbf{E}$

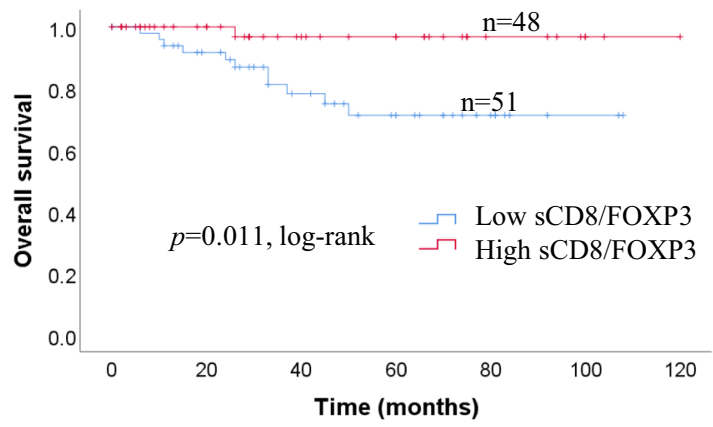

G

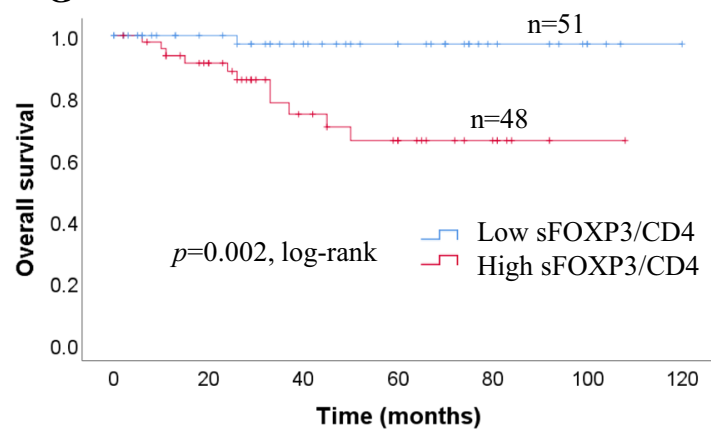

\section{Compliance with ethical standards}

Conflict of interest The authors declare no potential competing interests.

Open Access This article is licensed under a Creative Commons Attribution 4.0 International License, which permits use, sharing, adaptation, distribution and reproduction in any medium or format, as long as you give appropriate credit to the original author(s) and the source, provide a link to the Creative Commons licence, and indicate if changes were made. The images or other third party material in this article are 
included in the article's Creative Commons licence, unless indicated otherwise in a credit line to the material. If material is not included in the article's Creative Commons licence and your intended use is not permitted by statutory regulation or exceeds the permitted use, you will need to obtain permission directly from the copyright holder. To view a copy of this licence, visit http://creativecommons.org/licenses/by/4.0/.

\section{References}

1. Sorlie T, Tibshirani R, Parker J, Hastie T, Marron JS, Nobel A, et al. Repeated observation of breast tumor subtypes in independent gene expression data sets. Proc Natl Acad Sci USA. 2003;100:8418-23. https://doi.org/10.1073/pnas.0932692100.

2. Kim MJ, Ro JY, Ahn SH, Kim HH, Kim SB, Gong G. Clinicopathologic significance of the basal-like subtype of breast cancer: a comparison with hormone receptor and Her2/neu-overexpressing phenotypes. Hum Pathol. 2006;37:1217-26.

3. Mersin H, Yildirim E, Berberoglu U, Gulben K. The prognostic importance of triple negative breast carcinoma. Breast. 2008;17:341-6. https://doi.org/10.1016/j.breast.2007.11.031.

4. Janssen EM, Lemmens EE, Wolfe T, Christen U, von Herrath MG, Schoenberger SP. CD4+ T cells are required for secondary expansion and memory in CD8+ T lymphocytes. Nature. 2003;421:852-6. https://doi.org/10.1038/nature01441.

5. Galon J, Costes A, Sanchez-Cabo F, Kirilovsky A, Mlecnik B, Lagorce-Pages C, et al. Type, density, and location of immune cells within human colorectal tumors predict clinical outcome. Science. 2006;313:1960-4.

6. Sato E, Olson SH, Ahn J, Bundy B, Nishikawa H, Qian F, et al. Intraepithelial CD8+ tumor-infiltrating lymphocytes and a high CD8+/regulatory $\mathrm{T}$ cell ratio are associated with favorable prognosis in ovarian cancer. Proc Natl Acad Sci USA. 2005;102:18538-43.

7. Ashida A, Boku N, Aoyagi K, Sato H, Tsubosa Y, Minashi K, et al. Expression profiling of esophageal squamous cell carcinoma patients treated with definitive chemoradiotherapy: clinical implications. Int J Oncol. 2006;28:1345-52. https://doi.org/10.3892/ ijo.28.6.1345.

8. Nakano O, Sato M, Naito Y, Suzuki K, Orikasa S, Aizawa M, et al. Proliferative activity of intratumoral CD8(+) T-lymphocytes as a prognostic factor in human renal cell carcinoma: clinicopathologic demonstration of antitumor immunity. Cancer Res. 2001;61:5132-6.

9. Kawai O, Ishii G, Kubota K, Murata Y, Naito Y, Mizuno T, et al. Predominant infiltration of macrophages and CD8(+) T Cells in cancer nests is a significant predictor of survival in stage IV nonsmall cell lung cancer. Cancer. 2008;113:1387-95. https://doi. org/10.1002/cncr.23712.

10. Fukunaga A, Miyamoto M, Cho Y, Murakami S, Kawarada Y, Oshikiri T, et al. CD8+ tumor-infiltrating lymphocytes together with CD4+ tumor-infiltrating lymphocytes and dendritic cells improve the prognosis of patients with pancreatic adenocarcinoma. Pancreas. 2004;28:26.

11. Ali HR, Provenzano E, Dawson SJ, Blows FM, Liu B, Shah M, et al. Association between CD8+ T-cell infiltration and breast cancer survival in 12,439 patients. Ann Oncol. 2014;25:1536-43. https://doi.org/10.1093/annonc/mdu191.

12. Mahmoud SM, Paish EC, Powe DG, Macmillan RD, Grainge MJ, Lee AH, et al. Tumor-infiltrating CD8+ lymphocytes predict clinical outcome in breast cancer. J Clin Oncol. 2011;29:1949-55. https://doi.org/10.1200/JCO.2010.30.5037.

13. Matkowski R, Gisterek I, Halon A, Lacko A, Szewczyk K, Staszek $\mathrm{U}$, et al. The prognostic role of tumor-infiltrating CD4 and CD8 T lymphocytes in breast cancer. Anticancer Res. 2009;29:2445-511.
14. Aaltomaa S, Lipponen P, Eskelinen M, Kosma VM, Marin S, Alhava E, et al. Lymphocyte infiltrates as a prognostic variable in female breast cancer. Eur J Cancer. 1992;28A:859-64.

15. Hori S, Nomura T, Sakaguchi S. Control of regulatory $T$ cell development by the transcription factor Foxp3. Science. 2003;299:1057-61. https://doi.org/10.1126/science.1079490.

16. Sakaguchi S, Miyara M, Costantino CM, Hafler DA. FOXP3+ regulatory $\mathrm{T}$ cells in the human immune system. Nat Rev Immunol. 2010;10:490-500. https://doi.org/10.1038/nri2785.

17. Bohling SD, Allison $\mathrm{KH}$. Immunosuppressive regulatory $\mathrm{T}$ cells are associated with aggressive breast cancer phenotypes: a potential therapeutic target. Mod Pathol. 2008;21:1527-32. https://doi. org/10.1038/modpathol.2008.160.

18. Adams S, Gray RJ, Demaria S, Goldstein L, Perez EA, Shulman $\mathrm{LN}$, et al. Prognostic value of tumor-infiltrating lymphocytes in triple-negative breast cancers from two phase III randomized adjuvant breast cancer trials: ECOG 2197 and ECOG 1199. J Clin Oncol. 2014;32:2959-66.

19. Denkert C, von Minckwitz G, Darb-Esfahani S, Lederer B, Heppner BI, Weber KE, et al. Tumour-infiltrating lymphocytes and prognosis in different subtypes of breast cancer: a pooled analysis of 3771 patients treated with neoadjuvant therapy. Lancet Oncol. 2018;19:40-50.

20. Wolff AC, Hammond MEH, Allison KH, Harvey BE, Mangu $\mathrm{PB}$, Bartlett JMS, et al. Human epidermal growth factor receptor 2 testing in breast cancer: American Society of Clinical Oncology/College of American pathologists clinical practice guideline focused update. Arch Pathol Lab Med. 2018;142:1364-82. https://doi.org/10.5858/arpa.2018-0902-SA.

21. Hendry S, Salgado R, Gevaert T, Russell PA, John T, Thapa B, et al. Assessing tumor-infiltrating lymphocytes in solid tumors: a practical review for pathologists and proposal for a standardized method from the international immuno-oncology biomarkers working group: Part 1: assessing the host immune response, TILs in invasive breast carcinoma and ductal carcinoma in situ, metastatic tumor deposits and areas for further research. Adv Anat Pathol. 2017;24(5):235-51. https://doi.org/10.1097/ PAP.0000000000000162.

22. Loi S, Drubay D, Adams S, Pruneri G, Francis PA, LacroixTriki M, et al. Tumor-infiltrating lymphocytes and prognosis: a pooled individual patient analysis of early-stage triple-negative breast cancers. J Clin Oncol. 2019;37:559-69. https://doi. org/10.1200/JCO.18.01010.

23. Marginean F, Rakha EA, Ho BC, Ellis IO, Lee AH. Histological features of medullary carcinoma and prognosis in triplenegative basal-like carcinomas of the breast. Mod Pathol. 2010;23:1357-63. https://doi.org/10.1038/modpathol.2010.123.

24. Kurozumi S, Matsumoto H, Kurosumi M, Inoue K, Fujii T, Horiguchi J, et al. Prognostic significance of tumour-infiltrating lymphocytes for oestrogen receptor-negative breast cancer without lymph node metastasis. Oncol Lett. 2019;17:2647-56. https ://doi.org/10.3892/ol.2019.9938.

25. Ibrahim EM, Al-Foheidi ME, Al-Mansour MM, Kazkaz GA. The prognostic value of tumor-infiltrating lymphocytes in triple-negative breast cancer: a meta-analysis. Breast Cancer Res Treat. 2014;148:467-76. https://doi.org/10.1007/s1054 9-014-3185-2.

26. Huang Y, Ma C, Zhang Q, Ye J, Wang F, Zhang Y, et al. CD4+ and $\mathrm{CD} 8+\mathrm{T}$ cells have opposing roles in breast cancer progression and outcome. Oncotarget. 2015;6:17462-78. https://doi. org/10.18632/oncotarget.3958.

27. Liu F, Lang R, Zhao J, Zhang X, Pringle GA, Fan Y, et al. CD8(+) cytotoxic T cell and FOXP3(+) regulatory T cell infiltration in relation to breast cancer survival and molecular subtypes. Breast Cancer Res Treat. 2011;130:645-55. https://doi.org/10.1007/ s10549-011-1647-3. 
28. Liu S, Lachapelle J, Leung S, Gao D, Foulkes WD, Nielsen TO. CD8+ lymphocyte infiltration is an independent favorable prognostic indicator in basal-like breast cancer. Breast Cancer Res. 2012;14:R48. https://doi.org/10.1186/bcr3148.

29. Rubbert A, Manger B, Lang N, Kalden JR, Platzer E. Functional characterization of tumor-infiltrating lymphocytes, lymph-node lymphocytes and peripheral-blood lymphocytes from patients with breast cancer. Int J Cancer. 1991;49:25-31. https://doi. org/10.1002/ijc.2910490106.

30. Macchetti AH, Marana HR, Silva JS, de Andrade JM, RibeiroSilva A, Bighetti S. Tumor-infiltrating CD4+ T lymphocytes in early breast cancer reflect lymph node involvement. Clinics (Sao Paulo). 2006;61:203-8.

31. Schmidt M, Weyer-Elberich V, Hengstler JG, Heimes AS, Almstedt K, Gerhold-Ay A, et al. Prognostic impact of CD4-positive $\mathrm{T}$ cell subsets in early breast cancer: a study based on the FinHer trial patient population. Breast Cancer Res. 2018;20:15. https:// doi.org/10.1186/s13058-018-0942-x.

32. DeNardo DG, Coussens LM. Inflammation and breast cancer. Balancing immune response: crosstalk between adaptive and innate immune cells during breast cancer progression. Breast Cancer Res. 2007;9:212. https://doi.org/10.1186/bcr1746.

33. Sakaguchi $\mathrm{S}$, Ono M, Setoguchi R, Yagi H, Hori S, Fehervari $\mathrm{Z}$, et al. Foxp3+ CD25+CD4+ natural regulatory $\mathrm{T}$ cells in dominant self-tolerance and autoimmune disease. Immunol Rev. 2006;212:8-27. https://doi.org/10.1111/j.0105-2896.2006.00427 .x.

34. Bates GJ, Fox SB, Han C, Leek RD, Garcia JF, Harris AL, et al. Quantification of regulatory T cells enables the identification of high-risk breast cancer patients and those at risk of late relapse. J Clin Oncol. 2006;24:5373-80. https://doi.org/10.1200/ jco.2006.05.9584.

35. Castaneda CA, Mittendorf E, Casavilca S, Wu Y, Castillo M, Arboleda $\mathrm{P}$, et al. Tumor infiltrating lymphocytes in triple negative breast cancer receiving neoadjuvant chemotherapy. World J Clin Oncol. 2016;7:387-94. https://doi.org/10.5306/wjco.v7.i5.387.

36. Kim MH, Koo JS, Lee S. FOXP3 expression is related to high $\mathrm{Ki}-67$ index and poor prognosis in lymph node-positive breast cancer patients. Oncology. 2013;85:128-36. https://doi. org/10.1159/000351473.

37. Peng GL, Li L, Guo YW, Yu P, Yin XJ, Wang S, et al. CD8(+) cytotoxic and FoxP3(+) regulatory $\mathrm{T}$ lymphocytes serve as prognostic factors in breast cancer. Am J Transl Res. 2019;11:5039-53.

38. Zhou Y, Shao N, Aierken N, Xie C, Ye R, Qian X, et al. Prognostic value of tumor-infiltrating Foxp3+ regulatory $\mathrm{T}$ cells in patients with breast cancer: a meta-analysis. J Cancer. 2017;8:4098-105. https://doi.org/10.7150/jca.21030.

39. Miyashita M, Sasano H, Tamaki K, Hirakawa H, Takahashi Y, Nakagawa $S$, et al. Prognostic significance of tumor-infiltrating CD8+ and FOXP3+ lymphocytes in residual tumors and alterations in these parameters after neoadjuvant chemotherapy in triple-negative breast cancer: a retrospective multicenter study. Breast Cancer Res. 2015;17:124-x. https://doi.org/10.1186/s1305 8-015-0632-x.

Publisher's Note Springer Nature remains neutral with regard to jurisdictional claims in published maps and institutional affiliations. 